\title{
Predicting quality of life among family caregivers of people with spinal cord injury having chronic low back pain in Nepal: a cross- sectional pilot study
}

\author{
Pasang Doma Sherpa ${ }^{1,2} \cdot$ Luppana Kitrungrote $^{1} \cdot$ Wipa Sae-Sia $^{1}$
}

Received: 4 March 2018 / Revised: 28 June 2018 / Accepted: 2 July 2018

(c) International Spinal Cord Society 2018

\begin{abstract}
Study design Descriptive cross-sectional study.

Objective This study aims to determine the factors predicting quality of life among Nepalese family caregivers of people with spinal cord injury (SCI) having low back pain.

Setting Eight districts of the Bagmati Zone of Nepal.

Methods Sixty-five family caregivers of people with SCI having chronic low back pain were recruited from January to March 2017. The factors examined as independent variables included monthly household income, daily caregiving hours, functional independence of people with SCI, low back pain intensity, and functional disability of family caregivers. The measures were the Family Caregivers' and Spinal Cord Injury Patients' Demographic Form, the Modified Barthel Index, the Pain Intensity Scale, the Oswestry Disability Index, and the World Health Organization Quality of Life-BREF Nepali version. A hierarchical multiple regression analysis was used to predict quality of life.

Results Functional independence of people with SCI, monthly household income, and functional disability of family caregivers with chronic low back pain could significantly predict quality of life at $41 \%$ (adjusted $R^{2}=0.41, F_{\text {change }}(3,59)=$ $11.02, p<0.01)$. The functional dependence of persons with SCI was the most powerful factor contributing to QoL of caregivers $(\beta=0.36, p<0.01)$ followed by monthly household income $(\beta=0.30, p<0.01)$ and caregivers' functional disability $(\beta=-0.28, p<0.01)$.

Conclusion The findings suggested that in order to improve the quality of life of family caregivers who have chronic low back pain while providing care for people with SCI during a long period, health professionals should strengthen the functional independence of the patients after discharge to reduce the functional disability of the caregivers. Financial support is needed for caregivers who have a low income.
\end{abstract}

\section{Introduction}

During the past decades, low back pain (LBP) has become a common symptom and a major concern in the productive age group in developed and developing countries [1]. In $90 \%$ of LBP patients, it is usually a benign symptom and improves in 6 weeks; however, the remaining $10 \%$ of

Luppana Kitrungrote

Luppana.k@psu.ac.th

1 Faculty of Nursing, Prince of Songkla University, Hat Yai, Thailand

2 Department of Nursing, Nepal Institute of Health Sciences, Kathmandu, Nepal patients do not recover and develop chronic LBP [2]. In the long-term care of people with spinal cord injury (SCI), most caregivers are non-professionals who lack knowledge of back safety and lifting techniques [3]. These may be the leading causes of LBP. Previous studies showed that a high prevalence of chronic LBP among SCI family caregivers ranged from 58 to $65 \%[4,5]$. LBP has the potential to limit social and daily activities due to functional and psychological problems that will cause degeneration in the quality of life (QoL) [6]. Several studies found that chronic LBP was negatively correlated with QoL of the family caregivers [7, 8]. In addition, family caregivers with high levels of LBP had a significantly lower QoL than those who had low levels of LBP [6].

QoL is the perception of an individual's position in life in the context of the culture and value systems in which they 
live and in relation to their goals, expectations, standards, and concerns [9]. In a caregiving context, previous evidence showed that demographics and health/illness of patients and caregivers and the environment were related to QoL of the caregivers. For example, several studies have shown that lower household income due to caregiving was associated with caregiver distress and low QoL [10-12]. In addition, decreased functional independence of disabled people was related to increased burden on caregivers and worse overall QoL [11, 13, 14]. Caregiving hours were significantly associated with burden and well-being among SCI caregivers [15]. Previous studies also found that QoL was significantly lower for individuals with functional limitations $[16,17]$. In particular, people with LBP with functional limitations may develop a disability that interferes with their QoL [18]. Therefore, monthly household income, functional dependence of persons with SCI, daily caregiving hours, pain intensity of the caregivers, and functional disability were selected as the predicting variables because of their associations with QoL.

However, the majority of the caregiving studies have been conducted in developed countries. These results may not be generalized to the QoL of SCI caregivers with LBP in Nepal because of the differences of individual care needs and sociocultural situations. In addition, the incidence of SCI in Nepal is expected to be as high as 600-3500 cases annually [19]. However, QoL research in Nepalese SCI caregivers with LBP is limited. Therefore, the purpose of this study was to examine predictive factors, including monthly household income, functional dependence of persons with SCI, daily caregiving hours, and the LBP intensity and functional disability of the caregivers on QoL among SCI caregivers.

\section{Methods}

\section{Participants and recruitment}

A cross-sectional design was developed. Ethical approvals were obtained from the institutional review boards of the concerned university in Thailand, the Nepal Health Research Council, and the Spinal Injury Rehabilitation Center of Nepal. The participants were selected based on the convenient sampling technique. The inclusion criteria were the primary family caregiver providing care to a person with SCI more than 3 months, aged more than 18 years, having perceived LBP for more than 3 months after assuming the caregiver role, able to understand Nepalese language, and an absence of history of spine disorder.

The first author contacted persons with SCI by telephone that were discharged to the community from the Spinal Injury Rehabilitation Center. Family caregivers of the person with SCI were then approached by telephone. Potential participants who met the inclusion criteria were informed of the purpose, procedures, and confidentiality of this study. Written informed consent was obtained from each participant involved in this study.

The total sample included 65 adult family caregivers of persons with SCI residing in the communities of eight districts of the Bagmati Zone in Nepal. Data were collected between January and March 2017. The participants were asked about a convenient place and time for an interview. Most of them participated in their home and some interviews were at the Spinal Injury Rehabilitation Center. For illiterate participants, the first author read out the questionnaire and marked their response as stated. The questionnaire was self-administered for literate participants. Approximately $30 \mathrm{~min}$ was required for completion of the questionnaires.

\section{Measures}

Permission for use was obtained for all copyrighted questionnaires, except for the World Health Organization Quality of Life (WHOQOL)-BREF Nepali version. All of the measures were translated into Nepali using the back translation process [20].

\section{Demographic characteristics of family caregivers and $\mathrm{SCl}$ patients}

Demographics were assessed using the Family Caregivers' and Spinal Cord Injury Patients' Demographic Form developed by the researchers. Caregiver data included age, gender, marital status, education, occupation, monthly household income, relationship to SCI patient, duration of caregiving, daily caregiving hours, physical caregiving activities, and physical environment barriers in the caring activities. The SCI patient data included age, gender, and type of SCI.

\section{Functional independence of persons with SCI}

Functional independence was evaluated by the Modified Barthel Index that measured the activities of daily living to reveal the degree of independence of the persons with SCI from any assistance. It covered ten items of functioning activities. The scores are distributed among these items: personal hygiene, bathing self ( 5 points each); feeding, stair climbing, dressing, bowel control, bladder control (10 points each); and mobility, transfer (15 points each). A total score is obtained by summing the points for each of the items. The total score of the Modified Barthel Index has a range of $0-100$ with higher scores indicating greater independence [21]. 


\section{LBP intensity}

The Pain Intensity Scale was used to measure the LBP intensity of the caregivers. It is a numerical scale ranging from 0 to 10 points, in which " 0 " means absence of pain and " 10 " means unbearable pain. Higher scores represent a higher intensity of pain.

\section{Functional disability of caregivers}

The Oswestry Disability Index was used to measure back pain of the caregivers that affects their ability to manage their activities of daily life in ten sections. Each section is scored on a $0-5$ scale, where a score of 5 represents the greatest disability. The index is calculated by dividing the summed score by the total possible score, which is then multiplied by 100 and expressed as a percentage where a score of $0 \%$ represents no disability and $100 \%$ represents a great deal of disability [22].

\section{Quality of life}

The WHOQOL-BREF Nepali version was used to assess QoL of caregivers. It consists of 26 questions that are categorized into four domains including physical health, psychological, social relationship, and environment. Each item is rated on a 5-item Likert scale with scores of 1-5. The scores of each domain are calculated by the summation of the score of each item in the domain divided by the number of items in each domain and eventually transformed into a $0-100$ scale. The score of the overall QoL ranges from 26 to 130, where higher scores represent a higher QoL [23].

\section{Data analysis}

The data were analyzed using the Statistical Program for Social Sciences (SPSS, IBM Statistics) version 20. Since all questionnaires were checked for completion, there were no missing data. Data normality was confirmed using skewness and kurtosis of the distribution. Descriptive statistics were used to summarize the demographics of the family caregivers and SCI patients. Correlations between the independent variables (monthly household income, daily caregiving hours, functional independence of SCI patients, LBP intensity of the caregiver, and functional disability) and the dependent variable (QoL) were analyzed followed by a hierarchical multiple regression analysis to evaluate the predictive factors. The confounding variables (LBP intensity and daily caregiving hours) were entered in the first step of the hierarchical multiple regression. This was followed by entering monthly household income, functional disability of the caregiver, and the functional independence of the SCI patient in the second step. The assumptions of multiple regression analysis, that included normality, linearity, homoscedasticity, multicollinearity, and autocorrelation, were tested and they met the assumption tests. The significance level of the statistical test was 0.05 .

\section{Results}

Among 65 SCI caregivers with LBP, 55 (84.6\%) were female with a mean age of 35 years $(\mathrm{SD}=12.3$ years). Fifty-three $(81.5 \%)$ were married and $27(41.5 \%)$ had no education. Forty $(61.5 \%)$ of the 65 caregivers were wives. Fifty-three $(81.5 \%)$ caregivers were working (e.g., farmer and employee) while taking care of their SCI patients. The mean monthly household income was 13,224 Nepali rupee $(\mathrm{SD}=7820.3$ rupee $)(128.40 \mathrm{USD})(\mathrm{SD}=75.90 \mathrm{USD})$. The mean duration of caregiving was 14.8 months ( $\mathrm{SD}=$ 12.9 months) and the average daily caregiving hours was $14.7 \mathrm{~h}(\mathrm{SD}=7.7 \mathrm{~h})$. The common physical caregiving activities were lifting and transferring (98.5\%), bathing (93.8\%), and elimination care (92.3\%). Sixty percent of the caregivers had no caregiver assistant. More than half of the caregivers $(56.9 \%)$ reported physical environment barriers in caregiving activities (e.g., a narrow path for wheelchair, cramped space in room, and steep stairs). Among the SCI patients, $56(86.2 \%)$ were male with a mean age of 33 years $(\mathrm{SD}=9.7$ years) and $26(40 \%)$ were tetraplegic.

The mean score of functional independence of the SCI patients was $19.83(\mathrm{SD}=21.92)$. The mean scores of LBP intensity and functional disability of the caregivers were 5.0 $(\mathrm{SD}=1.6)$ and $33.02(\mathrm{SD}=12.41)$, respectively. The average QoL score of the caregivers was 71.22 ( $\mathrm{SD}=$ 12.51). The results of the correlational analysis of the QoL variable are displayed in Table 1.

Table 2 presents the results of the hierarchical multiple regression analysis to predict QoL. In the first model, QoL was tested simultaneously on the LBP intensity and daily caregiving hours of the caregiver. These two variables could significantly explain QoL (adjusted $R^{2}=0.13, F_{\text {change }(2,62)}$ $=5.57, p<0.01$ ). In the second model, monthly household income, functional disability of the caregiver, and

Table 1 Correlations of QoL with predicting variables $(N=65)$

\begin{tabular}{ll}
\hline Independent variables & QoL \\
\hline Monthly household income & $0.38^{* *}$ \\
Daily caregiving hours & $-0.34^{* *}$ \\
Functional independence of person with SCI & $0.54 * *$ \\
LBP intensity of caregivers & $-0.29 * *$ \\
Functional disability of caregivers & $-0.44^{* *}$ \\
\hline
\end{tabular}

$* * p<0.01$ 
Table 2 Predictors of QoL $(N=65)$

\begin{tabular}{|c|c|c|c|c|c|c|c|c|}
\hline \multirow[t]{2}{*}{ Model } & \multirow[t]{2}{*}{ Predictor } & \multirow[t]{2}{*}{ Unstandardized B } & \multirow{2}{*}{$\begin{array}{l}\text { Coefficients } \\
\text { SE }\end{array}$} & \multirow{2}{*}{$\begin{array}{l}\text { Standardized coefficients } \\
\text { beta }\end{array}$} & \multirow[t]{2}{*}{$t$} & \multirow[t]{2}{*}{$p$ value } & \multicolumn{2}{|c|}{$95 \% \mathrm{CI}$ of $\mathrm{B}$} \\
\hline & & & & & & & Lower & Upper \\
\hline \multirow[t]{2}{*}{ Model 1} & LBP intensity of caregivers & -1.45 & 0.90 & -0.19 & -1.61 & 0.11 & -3.26 & -0.35 \\
\hline & Caregiving hours & -0.56 & 0.19 & -0.35 & -0.29 & 0.00 & -0.94 & -0.18 \\
\hline \multicolumn{9}{|c|}{ Model 1: $R^{2}=0.15$, adjusted $R^{2}=0.13, F_{\text {change }(2,62)}=5.57, p<0.01$} \\
\hline \multirow[t]{5}{*}{ Model 2} & LBP intensity of caregivers & -0.33 & 0.79 & -0.04 & -0.43 & 0.67 & -1.90 & 1.24 \\
\hline & Daily caregiving hours & -0.10 & 0.19 & -0.06 & -0.55 & 0.59 & -0.48 & 0.27 \\
\hline & Income & 0.00 & 0.00 & 0.30 & 3.05 & 0.00 & 0.00 & 0.00 \\
\hline & Functional disability of caregivers & -0.28 & 0.11 & -0.28 & -2.53 & 0.01 & -0.50 & -0.06 \\
\hline & Functional independence of person & 0.21 & 0.07 & 0.36 & 2.98 & 0.00 & 0.07 & 0.34 \\
\hline
\end{tabular}

Model 2: $R^{2}=0.46$, adjusted $R^{2}=0.41, F_{\text {change }(3,59)}=11.02, p<0.01$

functional dependence of the person with SCI were entered. Finally, three of five determinants of QoL (functional dependence of the person with SCI, functional disability of the caregiver, and monthly household income) significantly predicted QoL at a level of $41 \%$ (adjusted $R^{2}=0.41, F_{\text {change }}$ $(3,59)=11.02, p<0.01)$. In this model, the functional dependence of the person with SCI was the most powerful determinant contributing to QoL of the caregivers $(\beta=$ $0.36, p<0.01)$ followed by monthly household income $(\beta$ $=0.30, p<0.01)$ and functional disability of the caregiver $(\beta=-0.28, p<0.01)$.

\section{Discussion}

The functional independence of the person with SCI was an important predictor on QoL of a caregiver with LBP. This result was consistent with previous studies [11, 14, 24]. In this study, nearly half of the persons with SCI were tetraplegic who were severely impaired and highly-to-totally dependent on their caregivers for daily living activities which may require greater expenditures and more daily caregiving hours. The caregiving tasks might exceed a caregiver's feeling of just being tired that results in a lack of energy and opportunity for recreation or leisure activities [25]. Thus, caring for persons with SCI with low functional independence is associated with poor QoL of the caregivers.

In addition, earlier studies showed that the functional disability of an individual negatively determined their QoL $[14,16,17]$, which is similar to the finding of this present study. Caregivers had chronic LBP resulting from using substantial time and effort in providing physical care for persons with SCI with physical caregiving environment barriers and no caregiver assistant. They not only might suffer from pain and physical discomfort, but may also develop a functional disability in lifting a heavy weight, mobility, and social engagement. Therefore, functional limitations may reduce a caregiver's capacity to accomplish normal activities and may profoundly affect their QoL [16].

Monthly household income was also associated with QoL of caregivers [10-12], a result which was supported by this present finding. The majority of caregivers in this study had a low monthly income, which was perceived as inadequate. Moreover, most persons with SCI were male and were totally or severely dependent on others for activities in their daily lives. Since males are considered the bread-winners of a family in Nepal, it is obvious a family may face economic problems. Unlike some developed countries, the SCI people in Nepal are deprived of sustainable financial support from the government and other organizations. Hence, the caregivers needed to use their own income to care for the disabled individual, which interfered with the family economic status [11]. Besides a reduced income, a caregiver faces greater SCI health expenses arising from specific care requirements of a dependent person with SCI [26]. Therefore, the monthly household income was related to QoL among caregivers.

In addition, although the daily caregiving hours and the LBP intensity of the caregiver did not predict QoL in a caregiver, the study findings should be cautiously considered in their homogeneities because of the small sample size which may limit generalization. These results were similar to the findings of previous studies [27, 28].

This study has some limitations. First, generalization of the results to other SCI caregivers with LBP should be cautiously applied because of the nonrandom sampling, small sample size, and recruitment of the caregivers of persons with SCI who had received care at our Spinal Injury Rehabilitation Center. Future research could replicate the study with a larger sample size of SCI caregivers from various settings. Second, only short-term prediction is useful, given that the regression analysis was conducted on 
cross-sectional data collected at a single time point. Therefore, no causal conclusion can be inferred. A longitudinal design would enhance the interpretation of causality. However, despite the limitations, this study has a notable strong point. This was the first research study in eight districts of the Bagmati Zone of Nepal to address QoL of SCI caregivers with LBP. The findings of this study provide baseline evidence to improve QoL of caregivers and for further research of QoL among SCI caregivers with LBP.

\section{Conclusion}

The findings offer insight into factors that predict QoL among SCI caregivers with LBP in Nepal. The influence of income, functional dependence of SCI patients, and functional disability of caregivers on QoL of caregivers can assist health professionals to improve QoL among SCI caregivers with LBP. Appropriate interventions should be developed to improve the functional independence of persons with SCI and decrease the functional disability of the caregivers. In addition, financial support should be provided to caregivers with low income who provide long-term care for persons with SCI in the community.

Acknowledgements The first author acknowledges Thailand's Education Hub for ASEAN Countries (THE-AC) and the Graduate School and Faculty of Nursing, Prince of Songkla University, Thailand for partially supporting the funding of this study. She also thanks the Spinal Injury Rehabilitation Center, Nepal and all of the participants who agreed to take part in this study.

\section{Compliance with ethical standards}

Conflict of interest The authors declare that they have no conflict of interest.

\section{References}

1. Mehrdad R, Shams-Hosseini NS, Aghdaei S, Yousefian M. Prevalence of low back pain in health care workers and comparison with other occupational categories in Iran: a systematic review. Iran J Med Sci. 2016;41:467-78.

2. Leboeuf-Yde $\mathrm{C}$, Lemeunier $\mathrm{N}$, Wedderkopp $\mathrm{N}$, Kjaer $\mathrm{P}$. Evidence-based classification of low back pain in the general population: one-year data collected with SMS Track. Chiropr Man Ther. 2013;21:30-37.

3. Tao H, McRoy S. Caring for and keeping the elderly in their homes. Chin Nurs Res. 2015;2:31-34.

4. Bardak AN, Erhan B, Gündüz B. Low back pain among caregivers of spinal cord injured patients. J Rehabil Med. 2012;44:858-61.

5. Pajeemas KN, Patpiya S, Preeda A, Siriwan SN. Factors associated with low back pain in non-professional caregivers of dependent spinal cord lesion patients. Int $\mathrm{J}$ Caring Sci. 2018;11:471-7.
6. Inoue $\mathrm{Y}$, Nagakura $\mathrm{H}$, Uesugi $\mathrm{M}$, Koeda $\mathrm{H}$, Naruse $\mathrm{S}$. The influence of low back pain on care workers' health-related quality of life. J Phys Ther Sci. 2011;23:603-6.

7. Neves EB, Pietrovski EF, Claudino RF. Quality of life and low back pain in primary caregivers of children with cerebral palsy. Cad Saúde Coletiva. 2015;23:50-56.

8. Schaller A, Dejonghe L, Haastert B, Froboese I. Physical activity and health-related quality of life in chronic low back pain patients: a cross-sectional study. BMC Musculoskelet Disord. 2015;16:62-70.

9. World Health Organization. WHQOL: measuring quality of life. 2016. http://www.who.int/mental_health/media/68.pdf.

10. Jeong YG, Jeong YJ, Kim WC, Kim JS. The mediating effect of caregiver burden on the caregivers' quality of life. J Phys Ther Sci. 2015;27:1543-7.

11. Xie H, Cheng C, Tao Y, Zhang J, Robert D, Jia J, et al. Quality of life in Chinese family caregivers for elderly people with chronic diseases. Health Qual Life Outcomes. 2016;14:99-108.

12. Du J, Shao S, Jin GH, Qian CG, Xu W, Lu XQ. Factors associated with health-related quality of life among family caregivers of disabled older adults: a cross-sectional study from Beijing. Medicine. 2017;96:1-7.

13. Middleton JW, Simpson GK, De Wolf A, Quirk R, Descallar J, Cameron ID. Psychological distress, quality of life, and burden in caregivers during community reintegration after spinal cord injury. Arch Phys Med Rehabil. 2014;95:1312-9.

14. Lawang W, Horey DE, Blackford J. Family caregivers of adults with acquired physical disability: Thai case-control study. Int $\mathbf{J}$ Nurs Pract. 2015;21:70-77.

15. Gajraj-Singh P. Psychological impact and the burden of caregiving for persons with spinal cord injury (SCI) living in the community in Fiji. Spinal Cord. 2011;49:928-34.

16. Loos-Ayav C, Chau N, Riani C, Guillemin F. Functional disability in France and its relationship with health-related quality of life-a population-based prevalence study. Clin Exp Rheumatol. 2007;25:701-8.

17. Stefane T, Santos AM, Marinovic A, Hortense P. Chronic low back pain: pain intensity, disability and quality of life. Acta Paul De Enferm. 2013;26:14-20.

18. Horng YS, Hwang YH, Wu HC, Liang HW, Mhe YJ, Twu FC, et al. Predicting health-related quality of life in patients with low back pain. Spine. 2005;30:551-5.

19. Rahimi-Movaghar V, Sayyah MK, Akbari H, Khorramirouz R, Rasouli MR, Moradi-Lakeh M, et al. Epidemiology of traumatic spinal cord injury in developing countries: a systematic review. Neuroepidemiology. 2013;41:65-85.

20. Brislin RW. Back-translation for cross-cultural research. J Cross Cult Psychol. 1970;1:185-216.

21. Roth E, Davidoff G, Haughton J, Ardner M. Functional assessment in spinal cord injury: a comparison of the Modified Barthel Index and the 'adapted' Functional Independence Measure. Clin Rehabil. 1990;4:277-85.

22. Fairbank JC, Couper J, Davies JB, O'Brien JP. The Oswestry low back pain disability questionnaire. Physiotherapy. 1980;66:271-3.

23. World Health Organization. WHOQOL-BREF: introduction, administration, scoring and generic version of the assessment: field trial version, 1996. http://apps.who.int/iris/bitstream/handle/ 10665/63529/WHOQOL-BREF.pdf? sequence $=1$ \&isAllowed $=y$

24. Rattanasuk D, Nantachaipan P, Sucamvang K, Moongtui W. A causal model of well-being among caregivers of people with spinal cord injury. Pac Rim Int J Nurs Res. 2013;17:342-55.

25. Ünalan $\mathrm{H}$, Gençosmanoğlu $\mathrm{B}$, Akgün $\mathrm{K}$, Karamehmetoğlu Ş, Tuna H, Önes K, et al. Quality of life of primary caregivers of spinal cord injury survivors living in the community: controlled study with short form-36 questionnaire. Spinal Cord. 2001;39:318-22. 
26. Chou YC, Chiao C, Fu LY. Health status, social support, and quality of life among family carers of adults with profound intellectual and multiple disabilities (PIMD) in Taiwan. J Intellect Dev Disabil. 2011;36:73-79.

27. Manca A, Eldabe S, Buchser E, Kumar K, Taylor RS. Relationship between health-related quality of life, pain, and functional disability in neuropathic pain patients with failed back surgery syndrome. Value Health. 2010;13:95-102.

28. Nogueira PC, Rabeh SA, Caliri MH, Dantas RA. Health-related quality of life among caregivers of individuals with spinal cord injury. J Neurosci Nurs. 2016;48:28-34. 